\title{
Síndrome inflamatorio multisistémico asociado a COVID-19 en niños y adolescentes: un llamado al diagnóstico
}

\author{
Multisystem inflammatory syndrome associated with COVID-19 \\ in children and adolescents: a call for diagnosis
}

Rolando Ulloa-Gutiérrez ${ }^{1,4}$, Gabriela Ivankovich-Escoto ${ }^{2,4}$ y Marco A. Yamazaki-Nakashimada3,4

\begin{abstract}
'Servicio de Infectología. Hospital Nacional de Niños "Dr. Carlos Sáenz Herrera", Centro de Ciencias Médicas de la Caja Costarricense de Seguro Social. San José, Costa Rica. 2Servicio de Inmunología y Reumatología. Hospital Nacional de Niños "Dr. Carlos Sáenz Herrera", Centro de Ciencias Médicas de la Caja Costarricense de Seguro Social. San José, Costa Rica.

${ }^{3}$ Servicio de Inmunología Clínica. Instituto Nacional de Pediatría. Ciudad de México, México.

${ }^{4}$ Red de Enfermedad de Kawasaki en América Latina (REKAMLATINA).
\end{abstract}

Fuentes de financiamiento: Ninguna.

No conflictos de interés de los autores.

Recibido: 28 de mayo de 2020

Palabras clave: COVID19; SARS-CoV2; síndrome inflamatorio multisistémico; niños; adolescentes.

Keywords: COVID19; SARS-CoV2; multisystem inflammatory syndrome; children; adolescents.

$\mathrm{E}$ n diciembre de 2019, un nuevo virus emergió de la ciudad de Wuhan, China, siendo capaz de producir enfermedad respiratoria aguda, desde presentaciones leves hasta fulminantes y principalmente en adultos. Posteriormente denominado el virus SARS-CoV-2 $2^{1}$, este es el agente causal de la pandemia actual COVID-19 declarada como tal por la Organización Mundial de la Salud (OMS) en marzo $2020^{2}$.

Desde el inicio de la pandemia, la mayoría de reportes de vigilancia epidemiológica y publicaciones médicas han documentado que, a nivel mundial, aproximadamente $97-98 \%$ de todos los casos de COVID registrados ocurren en adultos. Del restante porcentaje que ocurre en niños, la mayoría de ellos cursa con infección leve o asintomática. De aquellos que requieren hospitalización en salas de emergencia, salas de pediatría o unidades de cuidados intensivos pediátricos (UCIP), la mayoría se debe a dificultad respiratoria y neumonía.

No obstante, el 24 de abril de 2020, el Grupo de Estudio de Reumatología de la Sociedad Italiana de Pediatría emitió una alerta al gremio médico ante el incremento de casos de enfermedad de Kawasaki (EK) incompletos o atípicos con mayor resistencia a la inmunoglobulina intravenosa (IGIV), tendencia hacia el síndrome de activación macrofágica (SAM) e ingreso a UCIP. En varios de estos niños, se pudo documentar mediante laboratorio evidencia de infección reciente por SARS-CoV2 o se contaba con la historia de contacto con familiares positivos para el virus. Dos días después, la Sociedad de Cuidados Intensivos Pediátricos del Reino Unido y el Colegio Real de Pediatría y Salud del Niño del Reino Unido emitieron un comunicado de alerta mediante sus redes sociales y páginas web, debido al incremento también en niños de todas las edades en Londres y otras regiones del Reino Unido, de cuadros clínicos con traslape de hallazgos de Kawasaki atípico y síndrome de shock tóxico (SST), principalmente ${ }^{3}$. Como hallazgos de interés en estos pacientes, aparte de su estado de shock hiperinflamatorio, éstos presentaban muchas manifestaciones gastrointestinales. Seguidamente, el 28 de abril de 2020, la Asociación Española de Pediatría realiza una alerta a los padres de familia ante el incremento de casos similares a los descritos en Italia y Reino Unido ${ }^{4}$. Con posterioridad a estos reportes, se empezaron a notificar los primeros casos en los Estados Unidos de América (E.U.A.) y sobre todo en el Estado de Nueva York ${ }^{5}$, donde hasta hoy el número de reportes ya supera los 200 casos sospechosos (datos no publicados).

\section{Correspondencia a: \\ Rolando Ulloa-Gutiérrez \\ rulloag@ccss.sa.cr}

rolandoug@gmail.com 
El día 1 de mayo de 2020, el Colegio Real de Pediatría y Salud del Niño del Reino Unido designó el nombre de esta nueva entidad como síndrome pediátrico multisistémico inflamatorio (PMIS o PIMS por sus siglas en inglés) y propuso una definición de caso para su reconocimiento oportuno $^{6}$. La misma consistía en la presencia de tres criterios: 1) niños con fiebre persistente, inflamación y evidencia de disfunción de uno o más órganos (shock, o compromiso cardiaco, respiratorio, renal, gastrointestinal o neurológico) más otros hallazgos, incluidos criterios parciales o totales para EK; 2) exclusión de cualquier otra causa microbiana; 3 ) la prueba de reacción de polimerasa en cadena (RPC) para SARS-CoV-2 puede estar positiva o negativa.

Después de esta definición, el Centro para el Control y Prevención de Enfermedades de de Enfermedades (CDC) de E.U.A. emitió el 14 de mayo una definición modificada de caso y modificó el término a $\mathrm{MIS}-\mathrm{C}^{7}$. $\mathrm{Al}$ día siguiente, la OMS define el nuevo nombre como síndrome inflamatorio multisistémico (SIM/MIS) en niños $\mathrm{y}$ adolescentes con COVID-19 ${ }^{8}$. Dicha definición primaria de caso aplica para niños y adolescentes de 0 a 19 años de edad que cumplan con los criterios de fiebre $\geq$ de 3 días, y 2 de los siguientes:

- Brote o conjuntivitis bilateral no supurativa o signos de inflamación mucocutánea (boca, manos o pies).

- Hipotensión arterial o shock.

- Hallazgos de disfunción miocárdica, pericárdica, valvular, o anormalidades coronarias (incluyendo hallazgos ecocardiográficos o aumento de troponina/ NT-proBNP.

- Evidencia de coagulopatía (por TP, TPT, elevación de Dímeros-D).

- Manifestaciones gastrointestinales agudas (diarrea, vómitos, o dolor abdominal).

$\mathrm{y}$

Elevación de marcadores de inflamación como velocidad de eritrosedimentación (VES), proteína C-reactiva (PCR), o procalcitonina.

$$
\mathrm{y}
$$

Ausencia de otra causa obvia de inflamación como sepsis bacteriana, o síndrome de shock tóxico estafilocóccico o estreptocóccico.

$$
\mathrm{y}
$$

Evidencia de COVID-19 (RT-PCR, test de antígeno o serología positiva), o contacto probable con pacientes con COVID-19.

La primera publicación médica de casos pediátricos provino de Inglaterra el 6 de mayo 2020, donde se describen los primeros 8 pacientes con SIM $^{9}$. En este reporte, los autores describen un aumento de casos en un lapso de 10 días a mediados de abril, de shock hiperinflamatorio, Kawasaki atípico, síndrome de shock por enfermedad de
Kawasaki (SSEK) y SST. A diferencia de la mayoría de niños con EK, todos ellos eran mayor de cinco años de edad, excepto un niño de 4 años. Estos pacientes tuvieron mucho síntoma gastrointestinal, todos progresaron a shock caliente vasopléjico refractario a volumen y requirieron de soporte inotrópico. Entre otros hallazgos, en la ecocardiografía un paciente presentó dilatación coronaria importante y otro, hiper-refringencia pericoronaria. Todos fueron tratados con IGIV y antimicrobianos, y 7 de los 8 egresaron de la UCIP entre el día cuatro y seis. Un paciente de 14 años falleció debido a arritmia, shock refractario y accidente cerebrovascular. El SARS-CoV2 fue confirmado en dos pacientes y en otros cuatro hubo contacto con familiares positivos.

La primera publicación de Italia al respecto proviene de investigadores del Hospital General de Bergamo ${ }^{10}$, en la cual los autores comparan retrospectivamente dos períodos de tiempo de casos pediátricos semejantes a enfermedad de Kawasaki ("Kawasaki-like"), del 1 de enero de 2015 al 17 de febrero de 2020, y del 18 de febrero al 20 de abril de 2020. En este segundo período, ellos documentan 10 niños con cuadros "Kawasaki-like" y de los cuales la mitad tuvieron presentación clínica clásica. Esto representó un incremento de 30 veces el número de casos respecto al período anterior. Comparativamente, las edades de ambos grupos fueron 3 (2-5 años) versus 7,5 (3-5 años), y solamente uno de los diez eran menores de cinco años. En todos los pacientes, el aumento de marcadores de inflamación aguda fue común, pero en todos los hemocultivos fueron estériles. La RT-PCR para SARS-CoV2 fue positiva en dos de ellos, la IgG positiva en $80 \%$, mientras que la IgM sólo en $30 \%$. El ecocardiograma fue anormal en $6(60 \%)$ de ellos, y 2 pacientes tuvieron aneurismas coronarios mayores de 4 $\mathrm{mm}$. Todos los pacientes fueron tratados con IGIV, metilprednisolona como terapia coadjuvante en ocho de ellos, ácido acetilsalicílico (AAS) en dos, e inotrópicos en dos.

Una publicación reciente analizó retrospectivamente 35 niños que se hospitalizaron con disfunción sistólica aguda ventricular izquierda o shock cardiogénico e estado de inflamación multisistémica ${ }^{11}$ en 12 hospitales de Francia y uno de Suiza entre el 22 de marzo y 30 de abril de 2020. La mediana de edad fue de 10 años, ninguno tenía cardiopatía de fondo. Se documentó infección por SARS-CoV2 en $31(88,5 \%)$ pacientes e historia de contacto reciente con familiares con cuadro viral respiratorio agudo en $37 \%$. Un 80\% tuvo síntomas gastrointestinales y 2 de ellos requirieron incluso laparotomía exploratoria por abdomen agudo. Un $83 \%$ fueron admitidos directamente a la UCIP, $80 \%$ de los pacientes estaban en shock cardiogénico y requirieron fármacos inotrópicos. El ecocardiograma documentó dilatación de las arterias coronarias en $17 \%$, pero no aneurismas en el primer ecocardiograma. No hubo fallecidos. 
tratamiento porque se requiere un mayor número de casos y ensayos clínicos controlados; pero lo cierto, es que esta entidad debe agregarse a la lista de causas de alteraciones coronarias en lactantes, niños y adolescentes febriles.

Necesitamos con urgencia datos prospectivos y proveniente de redes multicéntricas, debido a la menor frecuencia de COVID-19 en pediatría. Para ello, Latinoamérica cuenta con diferentes redes de investigación y vigilancia que pueden ser de extrema utilidad. Mientras tanto, los médicos clínicos a cargo de estos niños y adolescentes, deben conocer los criterios diagnósticos del nuevo síndrome y estar alertas, sobre todo en países con número de casos crecientes en la actualidad como Brasil, Chile, y Ecuador, donde podría verse la mayor parte de pacientes graves semanas posteriores a la infección por SARS-CoV2.

\section{Referencias bibliográficas}

1.- Organización Mundial de la Salud (OMS), ed. (11 de febrero de 2020). "Intervención del Director General de la OMS en la conferencia de prensa sobre el 2019-nCoV del 11 de febrero de 2020".

2.- Organización Mundial de la Salud (OMS), ed. (11 de marzo de 2020). "Alocución de apertura del Director General de la OMS en la rueda de prensa sobre la COVID-19 celebrada el 11 de marzo de 2020".

3.- https://picsociety.uk/news/pics-statementregarding-novel-presentation-of-multi-systeminflammatory-disease/

4.- $\quad$ https://www.aeped.es/noticias/informacion-lasfamilias-respecto-al-comunicado-interno-alertasobre-casos-shock-pediatrico

5.- https://www1.nyc.gov/assets/doh/downloads/ pdf/han/alert/2020/covid-19-pediatric-multisystem-inflammatory-syndrome.pdf

6.- https://www.rcpch.ac.uk/sites/default/ files/2020-05/COVID-19-Paediatricmultisystem- $\% 20$ inflammatory $\% 20$ syndrome-20200501.pdf

7.- https://emergency.cdc.gov/han/2020/han00432. asp

8.- https://www.who.int/news-room/commentaries/ detail/multisystem-inflammatory-syndrome-in- children-and-adolescents-with-covid-19

9.- Riphagen S, Gómez X, González-Martínez C, Wilkinson N, Theocharis P. Hyperinflammatory shock in children during COVID-19 pandemic. Lancet 2020; 395: 1607-8. doi: 10.1016/S01406736(20)31094-1 Epub 2020 May 7.

10.- Verdoni L, Mazza A, Gervasoni A, Martelli L, Ruggeri M, Ciuffreda M, et al. An outbreak of severe Kawasaki-like disease at the Italian epicenter of the SARS-CoV-2 epidemic: an observational cohort study. Lancet 2020; May 13 Online ahead of print doi: 10.1016/S01406736(20)31103-X.

11.- Belhadjer Z, Méot M, Bajolle F, Khraiche D, Legendre A, Abakka S, et al. Acute heart failure in Multisystem Inflammatory Syndrome in Children (MIS-C) in the context of global SARSCoV-2 pandemic. Circulation 2020; May 17. Online ahead of print. doi: $10.1161 /$ CIRCULATIONAHA.120.048360.

12.- DeBiasi R L, Song X, Delaney M, Bell M, Smith K, Pershad J, et al. Severe COVID-19 in children and young adults in the Washington, DC metropolitan region. J Pediatr 2020; May13. doi: 10.1016/j.peds.2020.05.007 Online ahead of print.

13.- Toubiana J, Poirault C, Corsia A, Bajolle F, Fourgeaud J, Angoulvant F, et al. Outbreak of
Kawasaki disease in children during COVID-19 pandemic: a prospective observational study in Paris, France. 2020 May 14. doi: https://doi.org/10.1101/2020.05.10.2009 7394.

14.- Deza Leon M P, Redzepi A, McGrath E, AbdelHaq N, Shawaqfeh A, Sethuraman U, et al. COVID-19 associated pediatric multi-system inflammatory syndrome. J Pediatric Infect Dis 2020; May 22 Doi:10.1093/jpids/piaa061. Online ahead of print.

15.- Gámez-González L B, Murata C, MuñozRamírez M, Yamazaki-Nakashimada M. Clinical manifestations associated with Kawasaki disease shock syndrome in Mexican children. Eur J Pediatr 2013; 172: 337-42. doi: 10.1007/s00431-012-1879-1.

16.- Gamez-González L B, Moribe-Quintero I, Cisneros-Castolo M, Varela-Ortiz J, MuñozRamírez M, Garrido-García M, et al. Kawasaki disease shock syndrome: unique and severe subtype of Kawasaki disease. Pediatr Int 2018; 60: 781-90. doi:10.1111/ped.13614.

17.- Ulloa-Gutiérrez R, Alphonse M P, Dhanranjani A, Yeung R S M. Kawasaki disease-associated cytokine storm syndrome. In: Cytokine Storm Syndrome. Cron RQ, Behrens EM editors. Springer 2019. pp 393-406 https://doi. org/10.1007/978-3-030-22094-5_23. 